\title{
Spectroscopic studies of fluorescent perylene dyes
}

\author{
LENNART B.-Å. JOHANSSON \\ Deparment of Physical Chemistry, University of Umeå, S-901 87 Umeå, Sweden
}

and

HeInZ LANGHaLs*

Institut für Organische Chemie der Universität München, Karlstrasse 23, D-8000 München 2, F.R.G.

(Received 16 September 1990; in final form 2 January 1991; accepted 7 January 1991)

\begin{abstract}
The lowest electronic transition of the fluorescent perylene dye bis-(3,5-di-tertbutylphenyl)perylene-3,4:9,10-biscarboximide has been investigated by means of linear dichroism (LD), circular dichroism (CD), and by steady-state and time-resolved fluorescence spectroscopic techniques. The absorption and emission transition dipoles were found to be parallel with one direction along the whole vibronic progression of the spectra and thus correspond to a single electronic transition. A Förster radius of $54.6 \AA$ was found for the dye in ethanol at $298 \mathrm{~K}$. LD and absorption spectra obtained for the two stereoisomers of the dye when solubilized in a lyotropic liquid crystalline phase and in unilamellar vesicles suggest that the dyes form aggregates, presumably of $\mathrm{J}$ type. $\mathrm{CD}$ experiments suggest that these are linear $\mathrm{J}$ aggregates.
\end{abstract}

\section{INTRODUCTION}

PERYLENE DYES, perylene-3,4:9,10-tetracarboxylic bisimides (1) have unusually high light and chemical stabilities and are used as technical pigments [1]. Their strong fluorescence in solution was reported much later than their innovation [2] mainly due to the bad solubility of these dyes. This problem could be overcome by the introduction of tert-butyl groups to aromatic substituents [3]. Among these, dye 2 is of interest for practical applications $[4,5]$ and can be obtained in both stereoisomers [5]. These dyes can be used as laser dyes [6-8], for fluorescent solar concentrators [9], highly sensitive analysis methods $[10,11]$ and fluorescence immunoassays [18]. It is therefore of interest to obtain more information about the chromophore and its intermolecular interactions.

\section{EXPERIMENTAL}

The dyes $2 \mathrm{a}$ and $\mathbf{2 b}$ were prepared and purified according to the literature [5]. 1,2-Dioleoyl-snglycero-phosphocholine (DOPC) was purchased from Avanti Polar Lipids Inc. (U.S.A.). Details concerning the preparation of unilamellar vesicles are given in [12]. The lyotropic nematic phase was composed of potassium laurate, $\mathrm{KCl}$ and $\mathrm{D}_{2} \mathrm{O}$ as it described in Ref. [13]. The macroscopical alignment of the liquid crystal was checked by observing the samples when placed between two crossed polarizers.

The steady-state fluorescence spectra and anisotropies were obtained using a SPEX Fluorolog 112 instrument (SPEX Ind., New Jersey, U.S.A.), equipped with Glan-Thompson polarizers. The spectral bandwidths were 5.6 and $2.7 \mathrm{~nm}$ for the excitation and emission monochromators, respectively. Further details concerning correction factors and the calculation of fluorescence anisotropies are given in Refs [13] and [14]. A PRA 3000 ststem (Photophysical Research Ass. Inc., Canada) was used for single-photon counting measurements of the fluorescence decay. The excitation source was a thyratron-gated flash lamp (model 510C, PRA) filled with deuterium gas and operated at about $30 \mathrm{kHz}$. The excitation and emission wavelengths were selected by interference filters (Omega/Saven AB, Sweden) centred at 479 and $550 \mathrm{~nm}$. The fluorescence emission was also observed above $550 \mathrm{~nm}$ through a long pass filter Schott KV 550 (Schott, F.R.G.). The maximum absorbance of all samples was kept below 0.08 which corresponds to a total concentration of less than $10^{-6} \mathrm{M}$.

* Author to whom correspondence should be addressed. 
<smiles>[R]N1C(=O)c2ccc3c4ccc5c6c(ccc(c7ccc(c2c37)C1=O)c64)C(=O)N([R])C5=O</smiles>

1

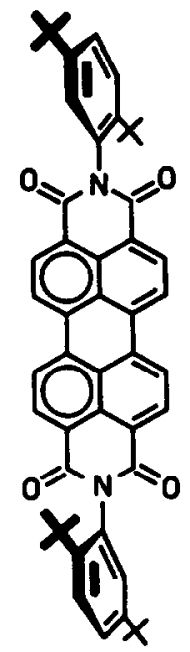

2a

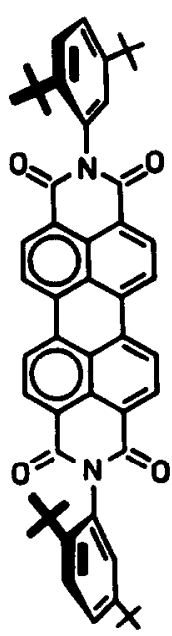

2b

Linear dichroism (LD) and circular dichroism (CD) were recorded on a JASCO J-600 (Japan). The absorption spectra were recorded on a Varian Cary 219 (U.S.A.) absorption spectrometer supplemented with sheet polarizers (HNP'B, Polarizers Ltd, U.K.).

\section{RESUlTS AND Discussion}

The absorption and fluorescence spectra of $\mathbf{2 a}$ and $\mathbf{2 b}$ are identical within experimental accuracy and furthermore, regarding their shape, similar to the spectra of perylene as can be seen in Fig. 1. However, compared to the perylene spectra they are red-shifted by about $100 \mathrm{~nm}$.

The fluorescence anisotropies of both isomers in propylene glycol were studied as a function of temperature and a typical result is displayed in Fig. 1. Clearly, both excitation and emission anisotropies are the same in the $440-640 \mathrm{~nm}$ wavelength region. Thus, the first electronic transition with its vibronic bands contains one direction of the electronic transition dipole moment. This is in accordance with the results found for perylene. With decreasing temperature the anisotropies increase below $253 \mathrm{~K}$ and reach a plateau value of 0.378 . This limiting anisotropy is slightly higher than that previously found for perylene and its alkyl derivatives, but it is the same value as found for the perylenoyl derivatives [14]. The limiting anisotropy was calculated as a mean value of the anisotropies determined in the range $430-650 \mathrm{~nm}$. The value is reproducible within \pm 0.003 . The

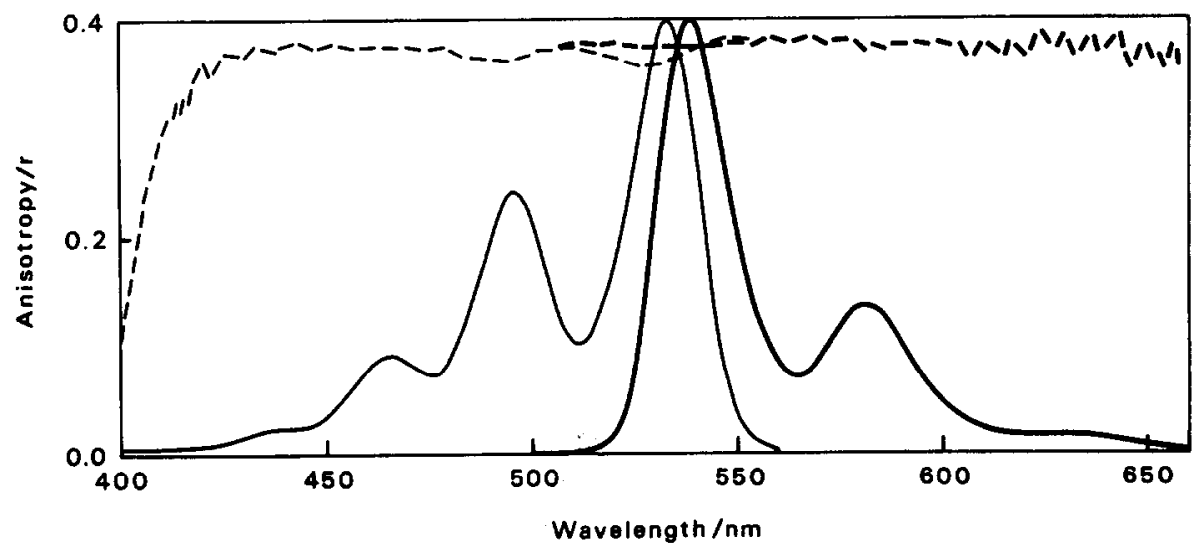

Fig. 1. Absorption (-) and fluorescence spectra (uncorrected) $(-)$ of $2 \mathbf{a}$ and $2 \mathbf{b}$ in propylene glycol. The excitation (---) and emission (--) anisotropies were determined at

$235 \mathrm{~K}$. The excitation and emission wavelengths were $533 \mathrm{~nm}$ and $540 \mathrm{~nm}$, respectively. 


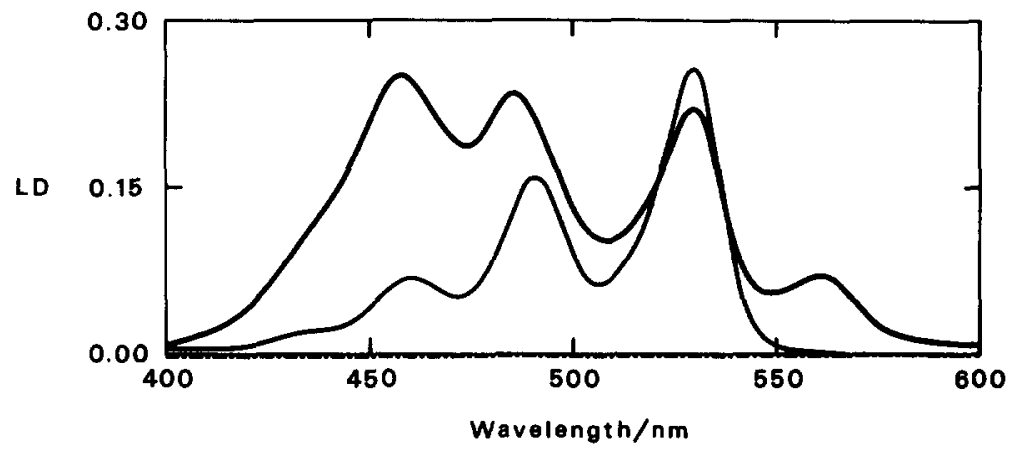

Fig. 2. Linear dichroism (LD) spectra of the perylene dyes $2 \mathbf{a}(\longrightarrow)$ and $2 \mathbf{b}(-)$ when solubilized in a lyotropic nematic liquid crystal which is macroscopically oriented.

value of 0.378 which we obtained is close to the theoretical maximum of 0.4 expected for parallel absorption and emission dipoles (for perpendicular vectors one would expect a value of -0.2 ). It can thus be concluded that these dipoles are nearly parallel for both isomers.

The anisotropy of $2 \mathbf{a}$ and $\mathbf{2 b}$ decreases monotonically from the maximum value of 0.378 below $245 \mathrm{~K}$ to 0.336 at $293 \mathrm{~K}$. Hence, at the lower temperatures the rotational motions are negligible on the timescale of fluorescence. We estimate a mean rotational rate of $3.3 \times 10^{7} \mathrm{~s}^{-1}$ at $293 \mathrm{~K}$. The corresponding rates [15] of perylene and 2,5,8,11-tetratert-butylperylene are $1.3 \times 10^{9}$ and $1.4 \times 10^{8} \mathrm{~s}^{-1}$. The much slower rotational rate of the isomers is qualitatively explained by the large difference in molecular volume.

The time-resolved fluorescence of $\mathbf{2 a}$ and $\mathbf{2 b}$ in 1,2-propanediol and methanol show mono-exponential decays with one and the same fluorescence lifetime of $3.8 \pm 0.1 \mathrm{~ns}$. The fluorescence quantum yield $\left(\phi_{\mathrm{f}}\right)$ in methanol is very close to one (cf. $\phi_{\mathrm{f}}=99 \%$ reported in Ref. [7]). Thus, the radiative lifetime of $2 \mathrm{a}$ and $2 \mathrm{~b}$ is also $3.8 \mathrm{~ns}$. As a comparison we note that the radiative and the measured lifetimes of perylene are $6.0 \mathrm{~ns}$ and $5.2 \mathrm{~ns}$ in ethanol [13]. The slightly longer radiative lifetime of perylene is compatible with its lower molar absorptivity $\left(\varepsilon_{\max } \simeq 40000 \mathrm{M}^{-1} \mathrm{~cm}^{-1}\right)$ for the first electronic transition as compared to $2 \mathrm{a}$ and $2 \mathrm{~b}\left(\varepsilon_{\max } \approx 95000 \mathrm{M}^{-1} \mathrm{~cm}^{-1}\right)$. The Förster radius $\left(R_{0}\right)$ of the isomers in ethanol has also been determined according to Ref. [12], yielding $R_{0}=$ $54.6 \pm 1 \AA$ at $298 \mathrm{~K}$. This number is similar to the typical $R_{0}$ values of rhodamine dyes, but smaller than the values found for perylene and its alkyl derivatives. For example, 2,5,8,11-tetra-tert-butylperylene has a Förster radius of $33 \AA$ [12].

Notice that $2 \mathrm{a}$ and $2 \mathrm{~b}$ differ only with respect to a rotation of the di-tert-butyl-phenyl ring about an $\mathrm{N}-\mathrm{C}$ bond. No spectroscopic differences could be detected within the accuracy of these experiments. This is in agreement with previous IR and UV-vis. studies concerning peak positions, line shape, molar absorptivity and fluorescence quantum yields [5].

The linear dichroism (LD) of $2 \mathbf{a}$ and $\mathbf{2 b}$ solubilized in a lyotropic nematic liquid crystal was also investigated. This liquid crystalline phase consists of long rod-like micelles that macroscopically orient in a strong magnetic field $(5.9 \mathrm{~T})$. The long axis of the aggregates align parallel to the magnetic field. The LD recorded with the linear polarizations of light parallel and perpendicular to the aggregates is shown in Fig. 2. The LD spectrum of $2 b$ has the same shape as the absorption spectrum $A(\lambda)$ in the $430-530 \mathrm{~nm}$ wavelength region. Hence, $L D(\lambda) / A(\lambda)$ is a constant which shows that this spectral region contains one direction of the electronic transition dipole moment. The average orientation of the electronic transition dipoles can be calculated using an order parameter [13] of $S=0.07$. This value is compatible with a low orientation and indicates that the long axis of the dye tends to orient parallel with the rod-like micellar aggregates, provided that the transition dipole moment is long-axis-polarized as in perylene. However from this experiment we cannot draw any conclusion as to whether the direction of the electronic transition dipole is parallel to the long or short axis of the molecular plane. For assigning this polarization one needs independent information about the orientation distribution of the molecules 


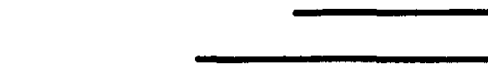

(20)

Fig. 3. Schematic linear orientation of dye molecules in $\mathbf{J}$ aggregates.

with respect to the long axis of the system. Taken together, the LD and the fluorescence anisotropy experiments show that the absorption spectrum between 420 and $550 \mathrm{~nm}$ is the lowest and a purely polarized electronic transition.

For $2 \mathrm{a}$ the absorption and the $\mathrm{LD}$ spectra are more complicated. Here, $\operatorname{LD}(\lambda) / \mathrm{A}(\lambda)$ is no longer constant with wavelength which strongly suggests that different kinds of absorbing species are present. The new band observed at $560 \mathrm{~nm}$ in both absorption and LD spectra indicates that aggregated forms of dyes are present.

These spectral changes are typical of the formation of $J$ type aggregates [16]. It is remarkable that the formation occurs even at concentrations of $10^{-6}-10^{-7} \mathrm{~mol} \mathrm{dm}^{-3}$ (but see Ref. [17]).

In order to find out whether the isomers mutually interact also in other aggregates of amphiphiles, we have solubilized them at different molar ratios in unilamellar vesicles of 1,2-dioleoyl-sn-glycero-phosphocholine (DOPC). At molar ratios of [DOPC]/[dye] $~$ $100-1000$ we observe absorption spectra for both dyes, where the $560 \mathrm{~nm}$ band is also present. The spectral shape is actually similar to that of the LD spectrum of 2a shown in Fig. 2. The shape of the fluorescent spectra changes so that more intensity is emitted at $\lambda_{\mathrm{em}} \simeq 560 \mathrm{~nm}$ with increasing molar ratio of [DOPC] $\left./ \mathrm{dye}\right]$. Thereby, the fluorescence spectra appear broadened. For such samples the excitation anisotropy (monitored at $536 \mathrm{~nm}$ ) is constant with wavelength, while the emission anisotropy (monitored at $536 \mathrm{~nm}$ ) strongly decreases at wavelengths longer than $560 \mathrm{~nm}$. We have also investigated the emission anisotropy when we excite at $490 \mathrm{~nm}$ where there is overlap between the different spectral species. The shape of the emission anisotropy is found to be identical with that observed at $\lambda_{\text {exc }}=530 \mathrm{~nm}$, but the magnitude of the anisotropy is about 0.03 lower. The change in spectral shape of the fluorescence spectra and the decreasing emission anisotropy observed at $\lambda_{\mathrm{em}}=560 \mathrm{~nm}$ are compatible with energy transfer to and from the monomer absorbing at $560 \mathrm{~nm}$.

Candidates for the new absorbing species are dimers, timers and $\mathrm{J}$ type aggregates which may give rise to absorption bands on the red and blue sides of the main vibronic band at $530 \mathrm{~nm}$. Additional information about the structure of such aggregates can be extracted from CD spectroscopy. We find no detectable CD effects in the $400-600 \mathrm{~nm}$ region. These results are compatible with a coplanar aggregation of the dyes where the transition dipoles are parallel, as is schematically illustrated in Fig. 3. If the aggregates are of $J$ type these can be linear (cf. Fig. 3) or helical. In order to find support for the helical structure, we have studied the $\mathrm{CD}$ of the isomers when solubilized in lipid vesicles which consist of optical active lipid molecules. The lipid matrix should induce a preference of one enantiomer of the two possible helices, and a CD effect of the absorption of the $J$ aggregates might be expected. No $C D$ effect was observed and this indicates that the $\mathrm{J}$ aggregates are linear as given in Fig. 3. Notice however, that this does not exclude that the aggregates are dimers or trimers.

\section{CONCLUSIONS}

The single electronic transition involving the long wavelength absorption of the perylene dyes and the parallel transition moment in absorption and emission make the perylene dyes an ideal probe for the investigation of orientation phenomena. The orientation can be controlled by the substituents at the terminal nitrogens. The results are important for example in the development of immunoliposomes [18, 19]. These results will be reported elsewhere. 
A further interesting result is that $2 \mathbf{a}$ forms aggregates more easily than does $\mathbf{2 b}$. One can compare this effect with the lower solubility of $\mathbf{2 a}$ in organic solvent compared to $\mathbf{2 b}$, both in solvents with high and low polarity [5]. This inidcates a higher crystal lattice energy of $2 a$ compared to $2 b$, because specific solvent effects can be exlcuded by the use of very different types of solvents. The lattice energy cannot be estimated by comparing the melting points, for the dyes decompose at temperatures above $360^{\circ} \mathrm{C}$ before melting. The higher lattice energy of $2 a$ corresponds to a more economic and simple packing of these molecules. A reason for this might be the point symmetric oriented tert-butyl groups in the molecule which gives the one side of the chromophore the same shape as the other one and provides a simple stacking structure.

Acknowledgements-Ihis work was supported by the Deutsche Forschungsgemeinschaft, Fonds der Chemischen Industrie and the Swedish Natural Science Research Council.

\section{REFERENCES}

[1] W. Herbst and K. Hunger, Industrielle Organische Pigmente, 1. Aufl., Verlag Chemie, Weinheim (1987).

[2] Hoechst AG (Inv. G. Geissler and H. Remy) D.O.S. 1.130 .099 (24 Oct. 1959): Chem. Abstr 57, P11346f (1962).

[3] H. Langhals, D.O.S. DE 3016764 A1 (30 Apr. 1980): Chem. Abstr. 96, 70417x (1982).

[4] A. Rademacher, S. Märkle and H. Langhals, Chem. Ber. 115, 2927 (1982).

[5] H. Langhals, Chem. Ber. 118, 4641 (1985).

[6] H.-G. Löhmannsröben and H. Langhals, Appl. Phys. B48, 449 (1989).

[7] E. M. Ebeid, S. A. El-Daly and H. Langhals, J. Phys. Chem. 92, 4565 (1988).

[8] M. Sadrai and G. R. Bird, Opt. Commun. 51, 62 (1984).

[9] H. Langhals, Nachr. Chem. Tech. Lab. 28, 716 (1980); Chem. Abstr. 95, R9816q (1981).

[10] H. Langhals, $Z$. Analyt. Chem. 320, 361 (1985).

[11] H. Langhals, Chem. Ind. (Düsseldorf) 37, 470 (1985).

[12] B. Kalman, L. B.-A. Johansson, M. Lindberg and S. Engström, J. Phys. Chem. 93, 8371 (1989); and Refs therein.

[13] L. B.-A. Johnasson, J. G. Molotkovsky and L. D. Bcrgelson, J. Am. Chem. Soc. 109, 7374 (1987); and Refs therein.

[14] L. B.-A. Johansson, J. Chem. Soc., Faraday Trans. 1 86, 2103 (1990).

[15] B. Kalman, N. Clarke and L.B.-A. Johansson, J. Phys. Chem. 93, 4608 (1989).

[16] Th. Förster, Naturwissenschaften 33, 166 (1946).

[17] E. Langhals and H. Balli, Helv. Chim. Acta 68, 1782 (1985).

[18] H. Langhals, II. Schott and R. A. Schwendencr, D.O.S. 3935257.9 (23 Oct. 1989).

[19] R. A. Schwendener, T. Trüb, H. Schott, H. Langhals, R. F. Barth, P. Groscurth and H. Hengartner, Biochim. Biophys. Acta 1026, 69 (1990). 